\title{
T-LEARNING VIA INTERACTIVE DIGITAL TELEVISION
}

\section{Andreja Samčović, Svetlana Čičević}

\author{
University of Belgrade \\ Faculty of Transport and Traffic \\ Engineering, \\ Belgrade, Serbia
}

\begin{abstract}
:
This paper presents the prospective use of interactive digital television (iDTV) in the process of learning via television (T-learning). The T-learning (learning by television) is reaching a considerable importance and is exploiting the available technologies of iDTV to allow opportunities for learning on every location and whenever users need. Following the description of T-learning opportunities and applications, the key elements of an interactive digital TV chain are described.
\end{abstract}

Key words:

T-learning, smart TV, digital TV, digital divide, IPTV.

\section{Acknowledgment}

This paper is partially supported by the Ministry of Education, Science and Technological Development of the Republic of Serbia (Projects No. 32025, 32048, 36022, and 36006).

\section{INTRODUCTION}

In the last forty years, television (TV) has shown a strong educational impact. Broadcast video content was stored on video cassettes and later on compact disks (CDs) and digital versatile disks (DVDs). With the introduction of digital television (DTV), new possibilities emerged for the use of television in education. DTV provides many technical improvements, such as, for example, better picture and sound quality. These properties are not the true contribution from the standpoint of education. The another promising potential of the digital TV is interactivity between the user and TV. Furthermore, additional content can be included in the broadcast signal, besides audio and video content (Crinon et al., 2006).

Probably the most essential reason for the introduction of digital techniques, as a prerequisite of the distribution of television signals, is a high degree of efficiency (efficient coding) in meaning the capacity of the transmission channel and control interference and noise. The broadcasters, as well as users, facilitate the process of reception, storage and content archiving. It is also important that the digital television (DTV) offers much greater flexibility in services compared with analogue television, because the available content is not limited to that what a producer wants to or wishes.
Correspondence:

Andreja Samčović

e-mail:

andrej@sf.bg.ac.rs

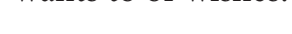


In DTV the broadcast sound and the image are digital, which have several advantages: simpler archiving, better use of frequency spectrum (within the same channel capacity the content amount is higher), better picture and sound quality, different services, supervised resistance against record interference and noise.

We gain the ability, as spectators, to have the influence on the content shown on our TV receivers. With the advent of this technology, the development of interactive content is not limited on the narrow professional circles and TV production houses. Viewers interaction with TV is a prerequisite for their active participation in the learning process. If the system is followed by the interaction of the viewer with $\mathrm{TV}$, the system can also offer to the viewer personalization of content. In the context of interactive digital television (iDTV), we understand the interaction and the use of remote control to request additional information on the broadcast program. This feature allows the selection of additional content (Atwere et al., 2003).

Depending on where the additional content is stored, interactivity can be divided into the local and global interactivity. In the local interactivity, the user can only access the content which is stored on the device called set-top-box (STB). On the other side, the global interaction takes place when a user accesses content over the return channel, which is stored by the content provider or a broadcaster. We are interested in interaction and associated additional related options offered by iDTV, making advantage of the most effective way of learning. While in the case with classical television the viewer can just watch a program and cannot interact, iDTV brings undoubtedly new potentials in education. The main advantage of television is that it is accessible to a wide audience, users accept TV technology as familiar and easy (Andrealis et al., 2007).

Interactive digital television combines two technologies: DTV and interactive applications. It can be used as media service in addition to voice and images. This technology allows to raise the level of interactivity between the viewer and the TV screen to the higher level. A viewer is no longer limited to a narrow set of functions (change channel, volume, switch on/off).

The television basically remains the medium that will be based on the video content. Video is the main advantage of television within learning process, differing from other electronic media. This is probably the main reason for implementation of television as a teaching tool. However, if only the video content was sufficient for the education process in the past, the introduction of electronic media has changed the situation.
The iDTV has a potential to become an important tool of informal learning through integration of education into the entertainment environment (edutainment), because of the familiarity of TV, easy utilization using the remote control and particularly, due to the power of upcoming interactive technologies. To be able to reuse this potential, it is necessary to develop new formats, applications and methodologies for delivery of educational content to target users.

The article is organized as follows: some aspects of T-learning are presented in the next section while the following part is dedicated to the digital divide. The description of the available technologies of iDTV used in $\mathrm{T}$-learning are discussed in the final part.

\section{T-LEARNING OPPORTUNITIES AND APPLICATIONS}

The term T-learning is defined as learning through interactive television, which refers to having interactive access to video-rich learning materials by a TV or other device that looks like a TV receiver with computer (Aarreniemi-Jokipelto, 2005). This device has to be reliable and easy to use (Lopez-Nores et al., 2004). The internet tools are often used to support virtual education. However, this scenario has a limitation, especially in the case of developing countries. TV penetration is much higher compared to internet worldwide. That is why new possibilities are explored for online education, now using television as a means to reach learners.

In recent years, there has been a considerable increase in the amount of educational content and support platforms for online learning or e-learning. This represents a final approach of citizenship education. The e-learning allows education to cheapen and facilitating access to everyone regardless of their geographical location and even temporary availability (Cassalino and D'Atri, 2005). The ability to access knowledge and training offered by high-level international experts is a fact which is highly enriching and democratizing. People with specific kinds of impairment in general, and persons with disabilities in particular, especially benefit from the new technologies. The persons with moving difficulties, disabled and those with problems in access to "conventional" education can enjoy this kind of learning.

Traditionally, analogue television offers viewers a passive attitude to the content features. The iDTV, however, give the opportunity to access the learning courses at study places, homes, school or work. Produced content for iDTV 
covers the interdisciplinary process, bringing together knowledge experts from various areas: computing, design, pedagogy, journalism, communication etc. Digital TV includes data and interactive applications in addition to transmitted audio-visual content (Piesing, 2006).

The general requirements for developing a T-learning course are the following: the interaction and communication must be feasible through the technology; the technology should support the the pedagogical content and the tasks presented to the students, as well as learners assesment; the possibility of content reusability through various subjects on different devices; and the opportunity to support learning for adults and elderly.

The aim of teaching and learning using digital TV promises to be of great value for education. The production of content oriented to T-learning is a challenge. The phase of pre-production stands for planning of requirements for a course available at iDTV platform. The challenge is to adapt the content with less text than a web page and to motivate the different audiences watching the television in the form of entertainment, for educational purposes. At the pre-production, there are three types of requirements: human, technological and pedagogical. It is noteworthy that the human and technological requirements can also be used for any application, such as e-government (T-government), T-commerce, T-health among others. However, teaching requirements are specific for the domain of T-learning.

\section{DIGITAL DIVIDE}

It should be remembered that there is a digital divide, which is not configured in only a technical problem of access or possession of technological tools, but in a much broader sense. It refers to some aspects related to each other: education, technological literacy, intellectual and practical skills of individuals, minorities and the disabled, technological innovation, the production of content, the quality of life, the expansion of specific communities, as well as the transition into the working world. It is easy to imagine that those who have access to the internet and know how to use it can be considered as more informed citizens, and more involved in social and political life. One aspect of digital divide can be the gap between those who have access to the internet and the information that can be found there and those who do not have such opportunities (www.elearningpapers.eu). Figure 1 represents internet use compared to age.

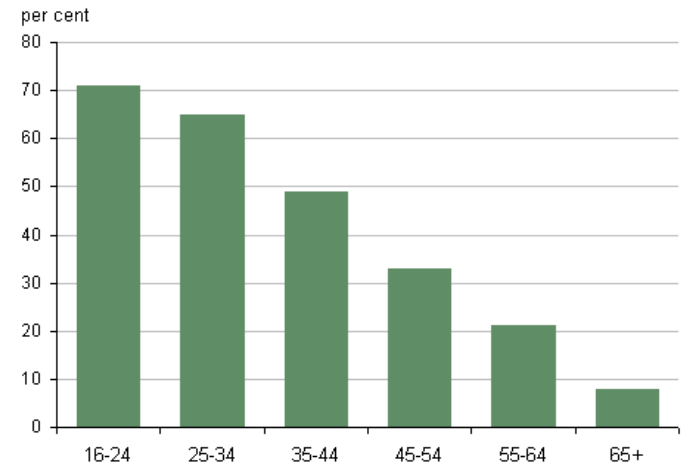

Fig.1. Internet use compared to age (Statistical bulletin: Internet access - households and individuals, 2011)

\section{HOW MUCH TV WE WATCH EVERY DAY}

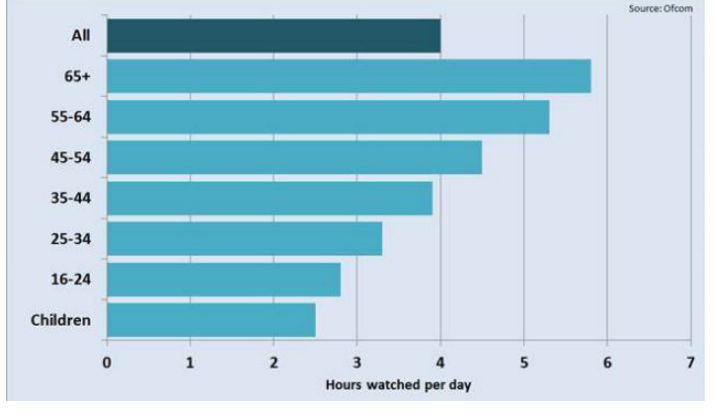

Figure 2. Television use compared to age (http://stakeholders.ofcom.org.uk)

Another possibility to overcome the digital divide is by using digital television. There are several benefits that digital communication provides to TV and one such benefit is interactivity. That means the ability of the user to interact with the elements displayed on the screen in unidirectional or bi-directional manner. The most important reason for useing digital TV in the context of learning is its accessibility both in a formal and informal manner. Television use compared to age is shown in Figure 2. We can see that older persons watch TV most compared to other age groups. Older persons and children can be the potential users of T-learning technology (Recchioni et al., 2007). On the other side, users of e-learning technology are predominantly younger students.

\section{INTERACTIVE SYSTEMS FOR T-LEARNING PLATFORM}

The realization of a T-learning platform requires from the content provider the planning and development of a specific architecture based on digital interactive tech- 
nologies. Technologies that can play a significant role in implementing T-learning platform are the following:

- Internet Protocol TV (IPTV);

- Video on Demand (VoD);

- Network Personal Video Recorder (NPVR).

\section{Internet Protocol TV (IPTV)}

Development and investment in interactive multimedia systems, such as IPTV system, have been increasing in the last years. When there is user interaction with the system, such services are called interactive services. If they wish to provide such services to users, service providers need to offer programs that will be broadcast according to the popularity of the content or they can offer a service in which content will be arbitrarily selected by the user. Interactive IPTV service will provide new users, and of course, more profit. In the development of interactive multimedia services, despite all benefits, many new technical problems must be resolved.

Specific interactive services that can be used via TV screens are already in use, while others are still being developed. Some of the more interesting interactive services that can already be used, or commercially expected in the near future are the following:

- IP video on demand;

- Internet browsing via the television;

- Notification of incoming calls on a television screen (showing caller ID), which the user can accept or reject;

- Creation a list of friends who are subscribers of different operators and correspondence with them;

- Information about the location or the currently selected TV channel of a friend;

- Sending invitations to friends to switch to the same channel;

- Transfer of paid content to a friend as a gift;

- Remote control for parents;

- Participation in the quiz with our friends;

- View to missed calls for mobile and fixed telephone lines;

- Electronic Program Guide;

- The possibility of skipping or selection of specific advertising.

Interactive characteristics allow many other things like skipping some TV ads, displaying only the desired. One can see the details of different events, buy from their own homes, book travel, etc., all with television. It is therefore clear that the boundaries between computers and television are increasingly disappearing. The development and installation of infrastructure that will enable all of these services and functions, of course, requires a lot of planning and effort. While most of the attention is devoted to what interactive multimedia system offers, little is said about how these systems will be compiled and at what price. Some technologies to support and guarantee the functioning of such systems already exist and are in function. However, it definitely needs more effort and extra work that would be actually able to talk about the functioning of the end-to-end system.

An interactive video system (e.g. IPTV) invoked many changes in the field of television. In traditional TV broadcasting systems, all channels are transmitted at the same time. The result is that sometimes the user has to choose between more interesting shows, because they are emitted at the same time. Because TV service providers do not want this scenario, interactive systems enable users to view favorite content to the user's personal schedule, i.e. when he wants to do. The user is thus able to not miss any of the content. The result includes more satisfied customers.

A set-top-box (STB) is a device based on IP protocol, which is used to connect IPTV head-end with a TV set, which is illustrated in Figure 3. The main function of a STB is to interpret and translate customer's requirements into IP messages sent to the head-end. These requirements represent the user's selection of specific content or services. In the response, STB will receive the encrypted content that needs to be decrypted and decoded in order to be properly presented on the TV.

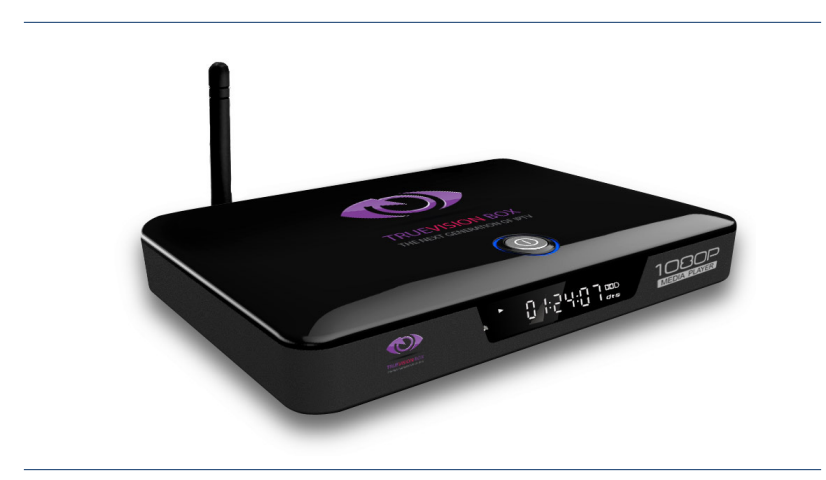

Figure 3. Set-top-box for IPTV

Video on Demand (VoD)

Video on Demand (VoD), as the most important IPTV interactive service system, offers to users a lot, but the most interesting is certainly the ability to play video content on 
demand. The service video on demand in the IPTV system allows users to select the video content and the time of its reproduction. This option currently represents a significant advantage compared to conventional television. The users of telecommunication services, due to faster lifestyles and increasing liabilities, less and less are able to watch a film, series and other audiovisual content in a pre-determined fixed terms.

Previous offer of classic television content is no longer adapted to the needs and desires of the user, i.e. it still offers the so-called linear audio-visual content. It is tailored to the way of life as twenty years ago. The way of life of today's users implies new demands. Today, there is no time to look at the content of the program schedule of the service provider. Therefore, there is a need to adapt the service provider program scheme to each individual user. VoD permits users to do so. This service is now also necessary because of the increase in the amount of available audio and video content. Therefore, due to the aforementioned reasons, it is necessary to develop smart system for storing, sorting, searching and playback of content, which is of importance in the process of Tlearning (www.iptv.com.au). One example of smart TV which can be used for VoD service is shown in Figure 4.

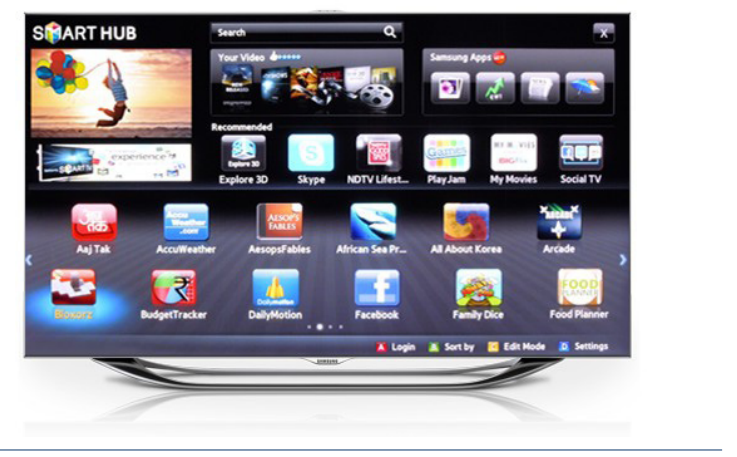

Figure 4. Smart TV (http://itcafe.hu/cikk)

Technically speaking, the provision of content that users can view on-demand over IP (Internet Protocol) network is somewhat more complex than the usual distribution of TV programs. Problems arise in providing content that our telecom provider can offer. The copyright in movies, shows, series is a key element in the implementation of the distribution system using new technologies, such as IPTV.

IPTV system can be broadly divided into two subsystems:

- LIVE TV system (channels that broadcast live);

- VoD systems (play on demand).
Network Personal Video Recorder (NPVR)

Network Personal Video Recorder (NPVR) is a technology that allows users to program a video recording and view the same content over at their own discretion. For a supply of NPVR, users specify in advance which programs provided by TV service with linear programming they want to save for later broadcast. A network provider copies the required content and archives them online. In fact, NPVR service is a type of VoD service, in which all of the content that has been identified by one or more users is recorded for later broadcast. One possible example of the NPVR is shown in Figure 5.

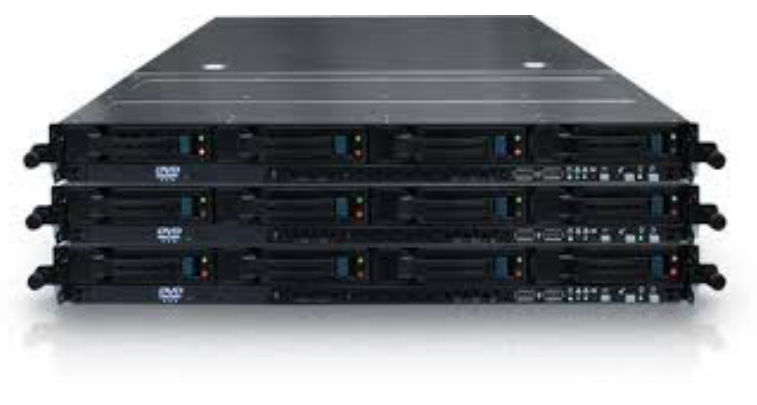

Figure 5. Network Personal Video Recorder (http://www.cs-sunnetwork.com/svod.html)

The watching rights over a NPVR can be different: one watch, more viewings, unlimited number of viewings. The program support for digital rights management is very important. The time shifted allows more flexibility in content viewing to end users. The content is recorded and stored inside a network and not on a user's device, which is the difference compared to classical video recorders. That possibility offers access to more advanced video services for users, and for operators to re-allocate storing capacities inside the network. Time shifted TV is a service which permits capture of live video streams and their storing in real time, which is important for a TV-learning system (Bijnens and De Gruyter, 2008).

Because in this case there are clear user requirements for the recording of certain content, provider has a fair view on the popularity of the content, so it can adapt optimal archiving strategy. Consumers can use a standard video recording controls, such as fast forward and rewind, pause and record. When NPVR platform is deployed, the desired content of subscribers is stored on the central server, which eliminates the need for expensive STB hard disk. 
There are several different approaches in implementing NPVR:

- Hosted NPVR space - provides to subscribers a space on the hard disk of VoD server. The space on hard disk varies between operators. When there is a request by IPTV interface, software captures certain content (content sections), whereby the recorded content is stored on hard disk server where it will be available to the subscriber.

- Recording of linear channels - another approach involves taking of a linear program as a whole and making it available to users in a fixed period of time, usually for a week.

\section{FINAL CONSIDERATIONS}

Television is still the medium present in almost every household, and therefore more opportunities exist for learning at our homes in much more relaxed situations. A relatively new concept of T-learning, which means learning by using television, can be a challenging tool to complement distance learning and e-learning.

T-learning employs the advanced technologies of interactive digital television and it can exploit those techniques by invoking new needs and requirements. The important technologies involved in the process of $\mathrm{T}$ learning are discussed and described in this paper. These technologies are still evolving in order to support more efficiently T-learning and other interactive services for TV. Interactive digital television (iDTV) is a promising platform for education since the technology can reach a large number of people and provide users with computing and communication interactivity.

There still remains a lot of questions that need to be answered in the near future, such as: What are the approaches that different manufacturers are taking? Which new features will be improved? What are the market place realities? How many people will have smart TVs in the next few years, how many actually use them, and what opportunities are available for learning providers? In this context, we can conclude that the real potential of T-learning is promising.

\section{REFERENCES}

Aarreniemi-Jokipelto, P. (2005). T-learning model for learning via digital TV. In 16th EAEEIE Annual Conference on Innovation in Education for Electrical and Information Engineering (EIE). Lapperanta, Finlandia.
Andrealis, A., Baldo, D., Benelli, G., Daino, G.L. (2007). Towards iTV applications' portability across digital terrestrial television frameworks. In IEEE SoftCOM 2007 International Conference on Software, Telecommunications and Computer Networks.

Atwere, D. et al. (2003). Interactive TV: a learning platform with potential. London, UK: Learning and skills development agency.

Bijnens, H., De Gruyter, J. (2008). How learning through digital terrestrial television (DTT) can lead to an inclusive society: The T-learning in the state of Sao Paolo (Brasil). In Developing Innovative Visual Educational Resources for Students Everywhere DIVERSE 2008, 1-3 July 2008. Harlem, The Netherlands.

Casalino, N., D’Atri, A. (2005). E-learning vs IS education \& research. The LUISS university experience. In Proceedings of International Conference on methods and technologies for learning ICMTL 2005, 9-11 March 2005 (pp. 97-103). Palermo, Italy, Chorianopoulos, K., Lekakos, G. (2008). Introduction to Social TV: Enhancing the Shared Experience with Interactive TV. International Journal of HumanComputer Interaction, 24(2), pp. 113-120.

Crinon, R.J. et al. (2006). Data broadcasting and interactive television. Proceedings of the IEEE, 94(1), pp. 102-118.

Lopez-Nores, M. et al. (2004). A technological framework for TV-supported collaborative learning. In Proceedings of the 6th International Symposium on Multimedia Software Engineering ISMSE 2004.

Piesing, J. (2006). The DVB multimedia home platform (MHP) and related specifications. Proceedings of the IEEE, 94(1), pp. 237-247.

Recchioni, M., Casalino, N., Castello, V., Roscani, M. (2007). An innovative training system by digital terrestrial television: TSC-learning web-based education. In Proceedings of the WBE 2007, 14-16 March 2007. Chamonix, France.

Statistical bulletin: Internet Access - Households and Individuals (2011).

Vossen, G., Jaeschke, P. (2002). Towards a Uniform and Flexible Data Model for Learning Objects. In Proc. 30th Annual Conf. of the Int. Bus. School Computing Assoc. (IBSCA), pp. 99-129. Savannah, Georgia. www.elearningpapers.eu, No. 12 (February 2009).

http://stakeholders.ofcom.org.uk/market-data-research

http://iptv.com.au

http://itcafe.hu/cikk

http://www.cs-sunnetwork.com/svod.html 